\title{
Inclusive cross sections for pairs of identified light charged hadrons and single protons from Belle $e^{+} e^{-}$ annihilation data
}

\author{
C. Van Hulse*, \\ on behalf of the Belle Collaboration \\ University of the Basque Country - UPV/EHU \\ E-mail: cvhulse@mail.desy.de \\ R. Seidl \\ RIKEN BNL Research Center \\ E-mail: rseidlercf.rhic.bnl.gov
}

\begin{abstract}
Cross sections differential in the hadron energy on hadron-pair and single-proton production from positron-electron $\left(e^{+} e^{-}\right)$annihilation are presented. The data were collected with the Belle detector at the KEKB $e^{+} e^{-}$collider at a center-of-mass energy of around $10.58 \mathrm{GeV}$. The cross section for single-hadron production provides very clean information on spin-independent fragmentation functions, which describe the spin-independent hadronization of quarks into final-state hadrons. Results on single-proton cross sections are presented, complementing the earlier published results on charged single-pion and single-kaon cross sections. Single-hadron cross sections do, however, not allow to distinguish between favoured and disfavoured fragmentation. Measurements of hadron pairs where the hadrons are produced nearly back to back do provide sensitivity to favored and disfavored fragmentation. Cross sections for the production of pairs of pions and kaons produced in the same hemisphere, in opposite hemispheres, and irrespective of the hemispheres they are produced in are presented.
\end{abstract}

XXIV International Workshop on Deep-Inelastic Scattering and Related Subjects

11-15 April, 2016

DESY Hamburg, Germany

${ }^{*}$ Speaker. 


\section{Introduction}

The hadronization or fragmentation of quarks into final-state hadrons is a non-perturbative process from the strong interaction described by fragmentation functions. Data from positronelectron $\left(e^{+} e^{-}\right)$annihilation provide the cleanest channel for the study of fragmentation functions, since contrary to semi-inclusive deep-inelastic scattering and proton-proton collisions sensitive to hadronization, the only non-perturbative objects entering the cross section are the fragmentation functions. In $e^{+} e^{-}$annihilation, an electron and positron annihilate into a virtual photon or $\mathrm{Z}$ boson, which in turn creates a pair of quarks and antiquarks that subsequently fragment into finalstate hadrons. The study of fragmentation functions extracted from $e^{+} e^{-}$annihilation do not only allow to deepen the understanding of the strong interaction, but these functions extracted from $e^{+} e^{-}$ annihilation can because of their universality serve as input for the study of the nucleon structure in semi-inclusive deep-inelastic scattering and proton-proton collisions.

Various measurements in $e^{+} e^{-}$annihilation that are sensitive to different fragmentation functions have been performed. Azimuthal asymmetries providing access to the Collins fragmentation function, which describes the fragmentation of a transversely polarized quark into an unpolarized hadron, were extracted [1-4]. In combination with measurements from deep-inelastic scattering $[5,6]$, the Belle data were used for a first extraction of the transversity distribution, the distribution of transversely polarized quarks in a transversely polarized nucleon, and of the Collins fragmentation function [7]. In addition, azimuthal asymmetries providing access to interferencefragmentation functions, which describe the hadronization of a transversely polarized quark into two final-state hadrons, were measured [8]. In combination with data from semi-inclusive deepinelastic scattering [9], these measurements again offer access to the transversity distribution, this time in conjunction with the interference fragmentation function [10].

Charged single-pion and single-kaon spin-independent cross-section measurements have been performed by, among others, the Belle and Babar Collaborations [11,12]. These data provide the cleanest access to spin-independent fragmentation functions at medium energy (center-of-mass energy $\sqrt{s} \approx 10 \mathrm{GeV}$ ). They do not only provide information on the formation of pions and kaons from the hadronization of a quark, but through quantum chromodynamic evolution they also allow access to the fragmentation functions of gluons. However, they are not able to distinguish between fragmentation of quarks and of antiquarks. Also, they provide only a reduced flavour discrimination. Therefore, complementary information from semi-inclusive deep-inelastic scattering and proton-proton collisions is necessary. Another means of accessing flavour and quark-antiquark discrimination is through the study of hadron-pair cross sections. At leading order, the singlehadron cross section differential in the fractional hadron energy $z$ with respect to the quark energy is proportional to

$$
\frac{d \sigma\left(e^{+} e^{-} \rightarrow h X\right)}{d z} \stackrel{\llcorner O}{\propto} \sum_{q} e_{q}^{2}\left[D_{q}^{h}\left(z, Q^{2}\right)+D_{\bar{q}}^{h}\left(z, Q^{2}\right)\right]
$$

where $Q=\sqrt{s}, e_{q}$ is the quark electric charge and $D_{q(\bar{q})}^{h}\left(z, Q^{2}\right)$ represents the spin-independent fragmentation function describing the hadronization of a quark $q(\bar{q})$ into a hadron $h$. When studying hadron pairs $\left(h_{1}, h_{2}\right)$ produced from the quark and antiquark, the cross section differential in the 
respective fractional hadron energies $z_{1}$ and $z_{2}$ is at leading order proportional to

$$
\frac{d \sigma\left(e^{+} e^{-} \rightarrow h_{1} h_{2} X\right)}{d z_{1} d z_{2}} \stackrel{L O}{\propto} \sum_{q} e_{q}^{2}\left[D_{q}^{h_{1}}\left(z_{1}, Q^{2}\right) D_{\bar{q}}^{h_{2}}\left(z_{2}, Q^{2}\right)+D_{q}^{h_{2}}\left(z_{2}, Q^{2}\right) D_{\bar{q}}^{h_{1}}\left(z_{1}, Q^{2}\right)\right] .
$$

Thus, by measuring cross sections for hadron pairs, one gains sensitivity to favoured and disfavoured fragmentation functions. For example, oppositely charged pions can originate from the fragmentation of an up and anti-up quark, where the up (anti-up) quark fragments into a positively (negatively) charged pion or the up (anti-up) quark fragments into a negatively (positively) charged pion. The former case corresponds to pure favoured fragmentation, while the latter to pure disfavoured fragmentation. If, on the contrary, one selects same-sign pions, then a combination of favoured and disfavoured fragmentation from the up quark is accessed. This interpretation of the hadron-pair cross section is only valid for hadrons fragmenting from different quarks, and becomes more intricate at higher orders.

\section{Hadron-pair and single-hadron cross section measurements}

Data collected at a center-of-mass energy of $10.58 \mathrm{GeV}$ and to a smaller extent of $10.52 \mathrm{GeV}$ by the Belle experiment, located at the asymmetric $e^{+} e^{-}$KEKB collider in Tsukuba (Japan), were analysed in order to extract single-proton cross sections, as such complementing the existing singlepion and single-kaon cross-section measurements, as well as cross sections for pairs of pions, pairs of kaons, and pairs of pions and kaons, all with same-sign and opposite-sign charge combinations [13]. The cross sections are extracted differentially in fractional hadron energy, in 36 equidistant bins from 0.1 to 1.0 for the single hadrons and in $16 \times 16$ equidistant bins from 0.2 to 1.0 for the hadron pairs. In order to have a handle on the distinction between hadrons originating from the same quark and from the quark and antiquark, the hadron-pair cross sections are extracted for hadron pairs irrespective of their orientation, for hadron pairs produced in the same hemisphere and for hadron pairs produced in opposite hemispheres, where the thrust axis is used for the assignation of the hemisphere (see Ref. [13]). Indeed, hadrons originating respectively from the quark and antiquark are more likely to be produced in opposite hemispheres, whereas hadrons originating from the same (anti)quark are more likely to be produced in the same hemisphere.

The cross sections are obtained from the measured raw hadron yields to which a series of corrections are applied. First, the data are corrected for particle misidentification, which on average amounts to $10 \%$, but can reach up to $50 \%$ in certain kinematic regions. The correction is based on particle-identification information extracted mainly from experimental data and complemented with Monte-Carlo simulation data where limited by statistical imprecision. Subsequently, the hadron yields are corrected for limited detector and tracking resolution, as obtained from MonteCarlo simulated data. The obtained quantities are corrected for processes where there is no unique quark-antiquark pair produced, such as quantum electrodynamic processes where instead a tauantitau pair is created, which subsequently decays into hadrons, or two-photon processes creating an electron-positron pair and a quark-antiquark pair. For data collected at a center-of-mass energy of $10.58 \mathrm{GeV}$, the contribution from resonant $\Upsilon(4 S)$ production with subsequent decay into $B$ mesons is removed as well. The data are also corrected for the detector acceptance, a correction again based on simulation, as well as for initial-state photon radiation by the beam leptons. For the 


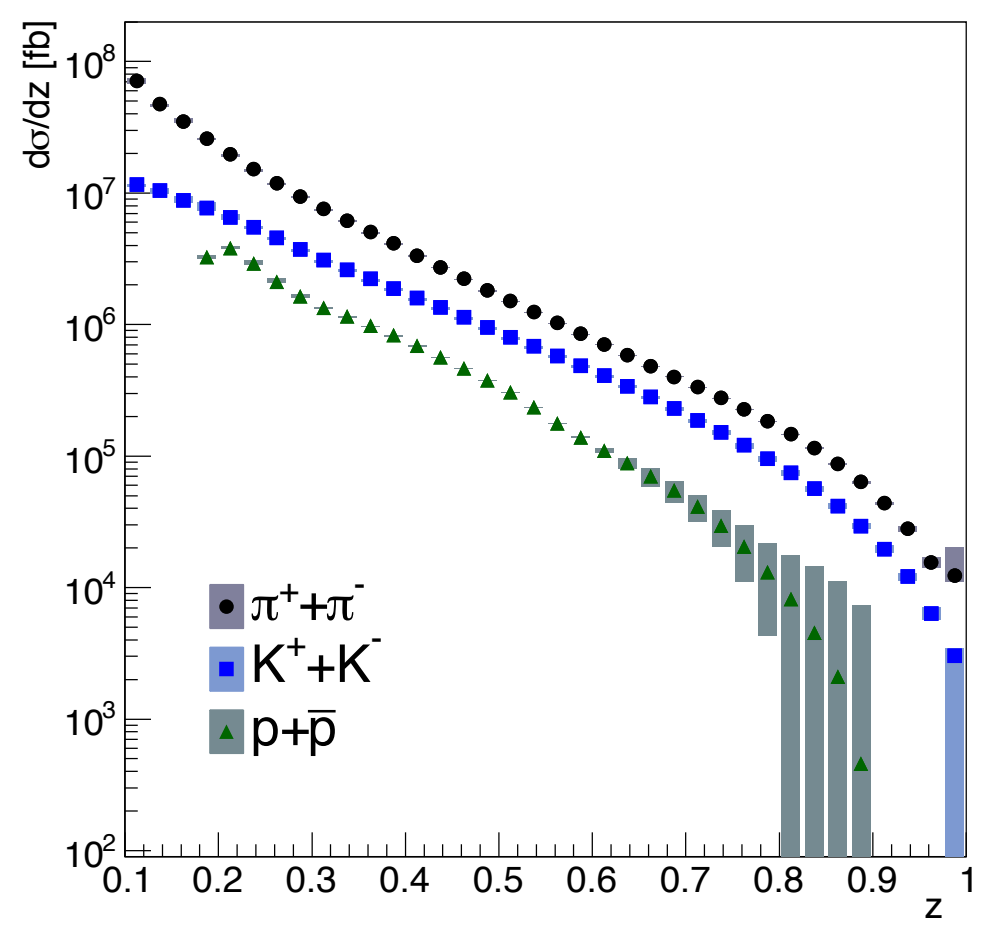

Figure 1: Charge-integrated pion (black circles), kaon (blue squares), and proton (green triangles) cross sections as a function of $z$. Statistical and systematic uncertainties are added in quadrature.

latter, the Monte-Carlo simulation is used in order to determine the fraction of hadrons originating from events where the radiated photon has an energy larger than $0.5 \%$ of $\sqrt{s} / 2$. In addition, there is a correction for weak decays provided. Since this is based on the Monte-Carlo simulation and given the associated uncertainty of the correction, the latter is only provided as an optional correction and not shown in the here presented results. The systematic uncertainty from each of the applied corrections is evaluated and constitutes the only systematic uncertainty besides the global uncertainty on track reconstruction and on the luminosity measurement; the sources of other possible systematic effects were found to be negligible. In all presented results, the systematic uncertainty is added in quadrature to the statistical uncertainty in order to form the total uncertainty.

\section{Results}

The result for the single-proton cross section differential in the fractional hadron energy is shown in figure 1, alongside with the pion and kaon cross sections. The latter are already published by the Belle Collaboration in Ref. [11] with finer binning, but are shown here for comparison. As seen, from the first bin at which protons are accessible, with $\langle z\rangle=0.1875$, up to a $z$ of 0.6 , the cross section is extracted with very high accuracy, while towards higher values of $z$ of around 0.8 and 0.9 , the results are dominated by the systematic uncertainties.

The cross sections for pairs of hadrons, irrespective of the hemisphere in which they are created, are shown in figure 2 for same-sign and opposite-sign charge combinations as a function of $z_{2}$ for various ranges in $z_{1}$. The cross section is largest for oppositely charged pion pairs. This 


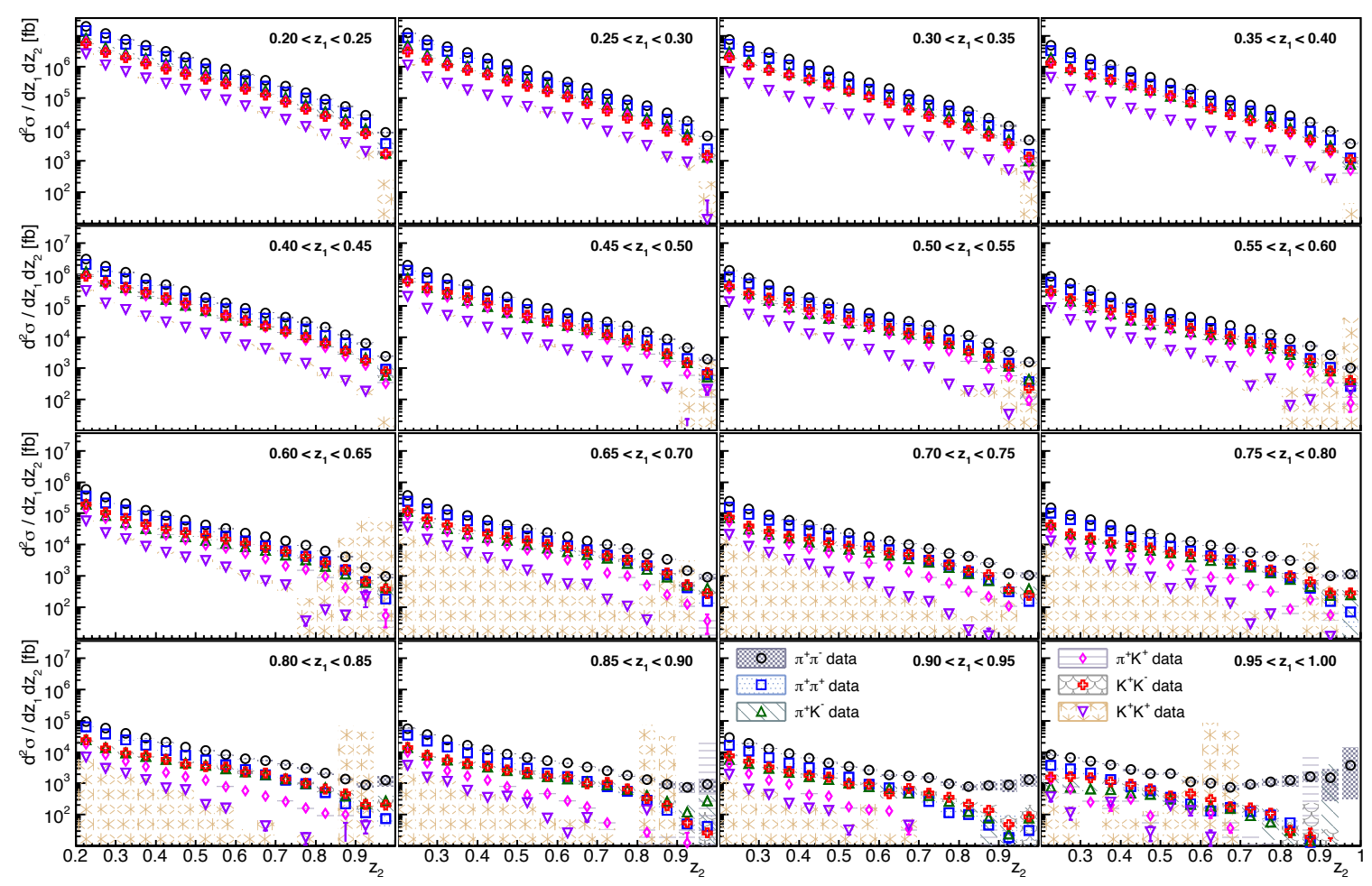

Figure 2: Cross sections for same-sign pairs of pions (blue squares), kaons (purple base-up triangles), and pion-kaon (magenta diamonds) and opposite-sign pairs of pions (black circles), kaons (red crosses), and pion-kaon (green base-down triangles) as a function of $z_{2}$ for different ranges in $z_{1}$, irrespective of the hemispheres in which the hadrons are produced. Statistical and systematic uncertainties are added in quadrature.

is consistent with up-quark dominance, the suppression of disfavoured fragmentation compared to favoured fragmentation, and the suppression of strange-quark production in the fragmentation process. At low values of $z$, the cross section for same-sign pion pairs is only slightly smaller than that for oppositely charged pion pairs, but falls off more rapidly with increasing $z$. In the LUND model [14], this can be interpreted as originating from the additional string break needed in disfavoured fragmentation compared to favoured fragmentation, and at larger values of $z$ there is less room for additional string breaks. The cross sections for oppositely charged kaon pairs and pion-kaon pairs are smaller than those for opposite-sign pion pairs and at low $z$ than those for same-sign pion pairs, which points to the suppression of strange-quark production in the fragmentation process. However, at larger values of $z$, the cross sections for oppositely charged kaon pairs and pion-kaon pairs become of the same size and even larger than that for same-sign pion pairs, which is again consistent with the suppression of disfavoured fragmentation and puts in perspective how much strangeness production is suppressed compared to disfavoured fragmentation. Also noteworthy is the observation that the same-sign kaon cross section is the smallest of all and falls off rapidly with increasing $z$, reflecting both the suppression of strangeness production and of disfavoured fragmentation. In order to cancel various systematic effects, the ratio of the cross sections for hadron pairs and oppositely charged pions is also extracted (not shown here). It can be found in Ref. [13]. Globally, the same observations as those for figure 2 can be made. 


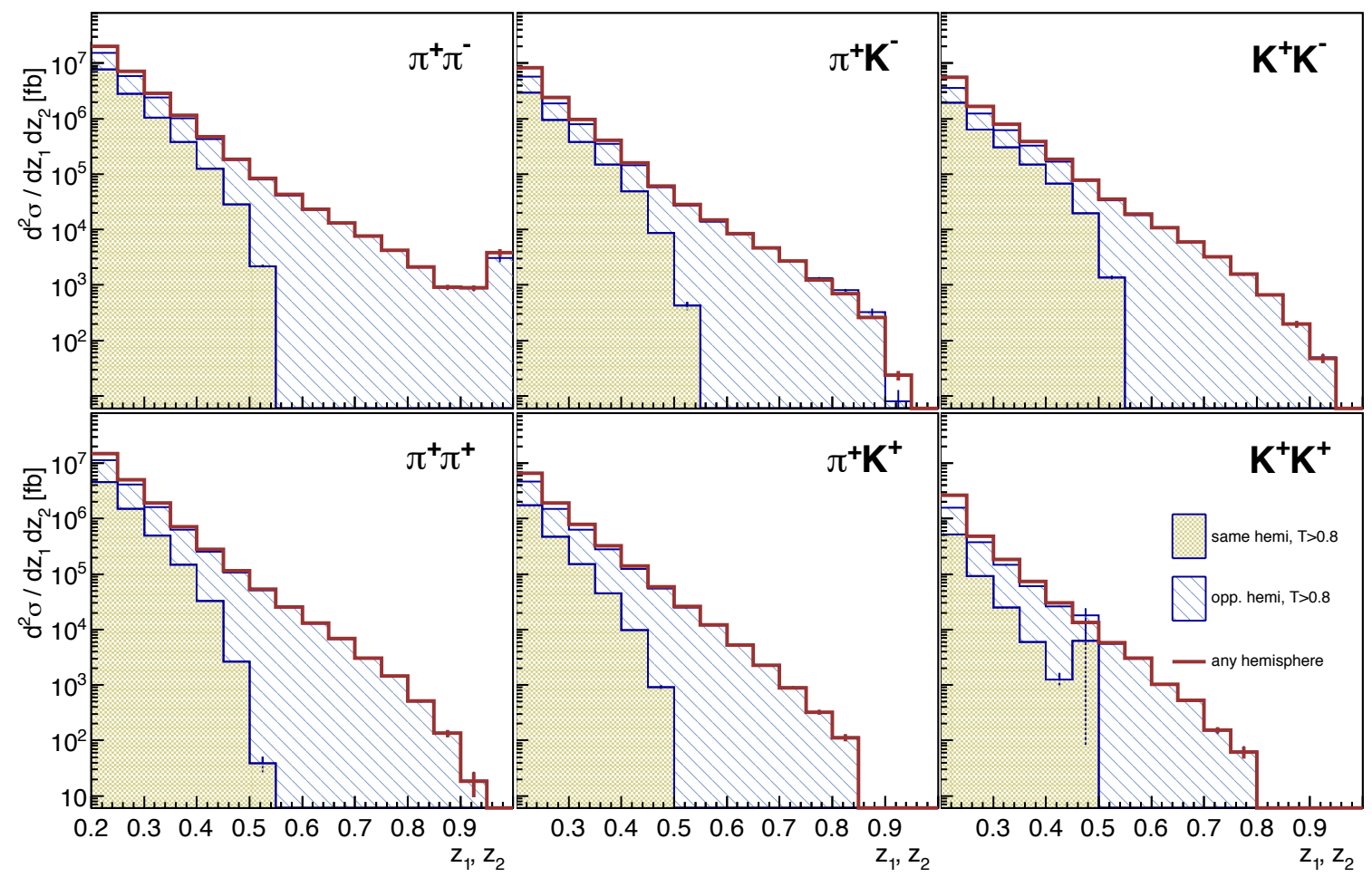

Figure 3: Cross sections for opposite-sign (upper row) and same-sign (lower row) pairs of pions (left column), pion-kaon (middle column) and kaons (right column), for hadrons produced in opposite hemispheres (blue, hatched histogram), the same hemisphere (beige, full histogram) and irrespective of the hemisphere (red line) as a function of $z_{1}=z_{2}$. For the cross sections for which the hadron-hemisphere configuration is not taken into account, a cut on the thrust value is not applied, while for the selection of a specific hemisphere configuration a lower cut of 0.8 is applied.

The hadron-pair cross sections for hadrons produced in the same hemisphere, in opposite hemispheres, and irrespective of their hemisphere are shown in figure 3 as a function of $z_{1}=z_{2}$. For $z_{1}=z_{2}$ above 0.5 , there are no hadron pairs produced in the same hemisphere. This is consistent with the picture that these hadrons are produced from the same quark and therefore the sum of their fractional energies cannot exceed one. A small amount of data above $z_{1}=z_{2}=0.5$ is understood to originate from the smearing of the thrust axis, reconstructed to assign the hemisphere of the hadrons, with respect to the initial quark-antiquark axis. From the figure it is again clearly seen that same-sign hadron pairs produced in opposite hemispheres fall off more rapidly with $z$ than their opposite-sign counter parts, indicating the suppression of disfavoured fragmentation.

In summary, the here presented results will contribute substantially to a better constraint of the proton fragmentation function. Based on their behaviour as a function of the fractional hadron energies, the cross sections for pairs of hadrons produced in the same hemisphere are consistent with the picture of hadrons originating predominantly from the same quark, while those for pairs of hadrons produced in opposite hemispheres are consistent with the picture of hadrons originating predominantly from different quarks. Therefore, the latter can help constrain single-hadron fragmentation functions, especially by providing sensitivity to quark-antiquark discrimination. 


\section{References}

[1] R. Seidl et al. [Belle Collaboration], Phys. Rev. Lett. 96 (2006) 232002.

[2] R. Seidl et al. [Belle Collaboration], Phys. Rev. D 78 (2008) 032011; Erratum-ibid 86 (2012) 039905.

[3] J. P. Lees et al. [BABAR Collaboration], Phys. Rev. D 90 (2014) 052003.

[4] Y Guan et al. [BESIII Collaboration], Int. J. Mod. Phys. Conf. Ser. 40 (2016) 1660033.

[5] A. Airapetian et al. [HERMES Collaboration], Phys. Lett. B 693 (2010) 11-16.

[6] E. S. Ageev et al. [COMPASS Collaboration], Nucl. Phys. B 765 (2007) 31.

[7] M. Anselmino et al., Phys. Rev. D 75 (2007) 054032.

[8] A. Vossen et al. [Belle Collaboration], Phys. Rev. Lett. 107 (2011) 072004.

[9] A. Airapetian et al. [HERMES Collaboration], JHEP 0806 (2008) 017.

[10] A. Bacchetta, A. Courtoy, and M. Radici, Phys. Rev. Lett. 107 (2011) 012001.

[11] M. Leitgab et al. [Belle Collaboration], Phys. Rev. Lett. 111 (2013) 062002.

[12] J. P. Lees et al. [Babar Collaboration], Phys. Rev. D. 88 (2013) 032011.

[13] R. Seidl et al. [Belle Collaboration], Phys. Rev. D. 92 (2015) 092007.

[14] Sjöstrand, Torbjorn, Comput. Phys. Commun. 82 (1994) 74. 